\title{
Research on the Evolutionary Game Path of Three Parties in Technology Finance from the Perspective of Project Risk
}

\author{
Guihuan YAN, Wei $\mathrm{LI}^{1}$ and Tingting GUO \\ Qilu University of Technology, Shandong Academy of Sciences, China
}

\begin{abstract}
The financing dilemma faced by technology enterprises has become an important obstacle to the further development of technology finance. This paper constructs the evolutionary game model of government, financial institutions and enterprises, introduces the project risk factor, and studies the cooperative path mechanism of multi-subjects in technology finance by combining stability analysis and SD simulation. The results show that: from the perspective of project risk, government subsidies to financial institutions are significantly better than subsidies to enterprises, and project risk plays a decisive role in tripartite cooperation. In addition to giving full play to the government's support to financial institutions, the project risks should be reasonably evaluated to minimize the information asymmetry between financial institutions and enterprises, so as to effectively solve the financing problems of technology enterprises.
\end{abstract}

Keywords. Technology finance, evolutionary game, stability analysis, SD simulation.

\section{Introduction}

At present, China's economy is in the critical period of "transforming mode and adjusting structure", and enterprise technological innovation has become the key to the highquality development of social economy in the future. In July 2020, the technology Innovation Board was successfully listed in the Shanghai Stock Exchange. In September 2021, The State Council approved the establishment of China's first corporate-based stock exchange, demonstrating the government's support for financing technology innovation. Technology financial as a series of financial tools, financial system, financial policy and systematic arrangement of financial service system [1], involving government, enterprises and financial institutions as the main body, past research has shown that governments often use their dominant position to promote cooperation between financial institutions and businesses, financial institutions as the main suppliers of funds, the risk of information asymmetry and earnings don't match, The lack of funds has become an important factor restricting the sustainable innovation of technology-based smes, and has affected the development of technology-based finance to a certain extent. Enterprise investment project risk is information asymmetry between partners and risk income does not match the decisive factor, but most scholars ignore it directly in the study, based on

\footnotetext{
${ }^{1}$ LI Wei, Master Degree Candidate, Qilu University of Technology (Shandong Academy of Sciences), 3501 Daxue Road, Changqing District, Jinan city, Shandong Province, China; E-mail: 1w1580@126.com.
} 
the evolutionary game matrix introduced in project risk factor, through the stability analysis and simulation of SD, and discuss the project risk horizon, technology path of win-win cooperation mechanism of financial tripartite main body.

In order to solve the financing dilemma of technology-based smes, many scholars focus on the cooperation mechanism of multi-stakeholder in technology finance. There is no unified definition of sci-tech finance in foreign countries, and there are few researches on cooperation mechanism. Relevant researches mainly focus on the evolution of government policies, the development of sci-tech enterprises, and the efficiency analysis and optimization of commercial financial system [2].In this paper, domestic literature is mainly sorted out. Yu Liping [3] points out that the active guidance of the government can significantly promote enterprise financing from the perspective of constructing the supporting system of technology finance and solving the financing problems of technology smes. Li Yuanyuan et al. [4] put forward the definition and construction of sci-tech finance network based on capital flow and information flow, and pointed out that the coordinated operation and risk prevention and control of sci-tech finance should not only rely on the government, but also other entities such as sci-tech enterprises and financial institutions should improve their own business models to achieve win-win cooperation. Wang Liming et al. [5], based on the life cycle theory, conducted a game analysis on the behavioral interaction between multiple stakeholders in the technology financial system in different life stages, and concluded that when multiple stakeholders are in a balanced state, innovation development can be significantly promoted. Long Xiaoyan et al. [6] put forward the cooperative service model between the government and financial institutions, and pointed out that the cooperation between the two parties can effectively promote the development of technology enterprises. Tan Jiajing et al. [7] and Yang Yi et al. [8] used evolutionary game model to analyze the cooperation stability between banks and technology enterprises. The latter introduced government reward and punishment mechanism on the basis of the original model, and concluded that when the penalty amount could make up for the loss caused by unilateral withdrawal, banks and technology enterprises would tend to have long-term stable cooperation. Yan Junmei et al. [9] analyzed the "absence" and "dislocation" of government and market in the process of financial support for tech enterprises from the perspective of supply side, so as to improve the support efficiency of tech finance.

From the above analysis, it can be seen that most domestic scholars focus on the cooperation mechanism between multiple stakeholders. For the financing difficulties of technology enterprises, most scholars realize that the root cause is the mismatch between benefits and risks, but there are few researches on the cooperation mechanism of technology and finance subjects based on project risk factors. Combined with the research and analysis of the existing literature, the research contributions of this paper are reflected in the following aspects: first, it builds an evolutionary game model for the three stakeholders of government departments, financial institutions and technology enterprises, and simulates the influence of government on the strategic choice of financial institutions and technology enterprises under different subsidy policies through SD simulation; The second is to introduce project risk coefficient innovatively and study the path mechanism of three parties under different risk coefficient; Third, the influence of key variables on system stability is further studied to build a more stable tripartite cooperation and win-win mechanism of technology and finance. 


\section{The Construction of Evolutionary Game Model}

\subsection{Subject Analysis}

As an important subject of technology finance, the government tends to provide support for enterprises' technology innovation, but limited financial funds determine that it is far from enough to rely on government power alone [10].As the main provider of funds, financial institutions will only provide funds to the customers who most meet the income target due to their huge loan demand groups, obviously most small and medium-sized technology enterprises are not included. At the same time, in order to seek development, enterprises will naturally try their best to find funds if their investment projects meet the risk and return expectations. However, the asset-light and high-risk characteristics lead most small and medium-sized technology enterprises into "financing dilemma". In order to solve this problem, the government usually guides financial institutions to cooperate with small and medium-sized technology enterprises. Due to the uncertainty of the risk of investment projects, the three parties in technology finance will play the game from the perspective of maximizing their own risk and return.

\subsection{The Basic Assumptions of the Model}

- Hypothesis 1: government departments, financial institutions and technology enterprises are all bounded rational people [11]. Government and technology enterprises are risk-neutral, while financial institutions are risk-averse. In the process of cooperation, they all play dynamic game with the goal of maximizing their own interests, but the strategic choices of the three parties will affect each other and form dynamic game relations.

- Hypothesis 2: The three parties in technology finance each have two choice strategies, that is, the government can choose "subsidy" and "no subsidy", with probabilities of $\lambda$ and $1-\lambda$ respectively. Financial institutions can choose to "invest" and "not invest" with probabilities of $x$ and $1-x$ respectively. Technology enterprises can choose "financing" and "not financing", with probabilities of $p$ and 1- $p$ respectively. $(0 \leq \lambda, x, p \leq 1)$

- Hypothesis 3: The government has two forms of subsidy. It can directly provide financial subsidies to technology enterprises or financial institutions, and the two ways can be carried out at the same time without affecting each other. On the premise of government "subsidy", when financial institutions choose "investment", no matter whether enterprises choose "financing", financial institutions can get government subsidy.

- Hypothesis 4: When a technology enterprise chooses "financing" and a financial institution chooses "no investment", the enterprise can choose to raise money from non-financial institutions at a higher capital cost. When the enterprise chooses "no financing", it means that the risk loss of the project itself exceeds expectations, and the enterprise will choose to give up the investment project.

- Hypothesis 5: The risks of technological innovation projects are all reflected by the expected loss of project investment failure .If the technology enterprise has obtained the investment of financial institutions, part of the loss faced by the enterprise will be transferred to the financial institutions, and the expected loss of the two is proportional, that is, the greater the risk of the technology 
enterprise project, the greater the risk of capital loss faced by financial institutions.

\subsection{Establish the Tripartite Evolutionary Game Model}

First of all, variables in the game model are explained: $C_{0}$ is the investment amount of scientific and technological innovation projects, $r^{\prime}, \xi$ are the subsidy coefficient of the government to financial institutions and enterprises respectively. In the implementation of the project, When the government chooses "subsidy", it gets $R g$, otherwise it gets $R g^{\prime}$.Financial institutions will fully consider the expected return and risk of investment when making strategic choice. $r_{0}$ is the interest rate of loans provided by financial institutions to technology enterprises, and $\beta$ is the risk loss coefficient faced by financial institutions. When the investment project cannot be supported by financial institutions, technology enterprises can only borrow money from non-financial institutions. $r$ is the basic interest rate of non-financial institutions. In addition, in order to pursue the maximization of interests, technology enterprises pay more attention to the value of the project itself while considering the cost of capital, that is, to evaluate the expected return $(\alpha)$ and risk $(v)$ loss coefficient of technology innovation projects. $k$ is the risk transfer coefficient. According to hypothesis 5 above, $\beta$ is directly proportional to $v$, that is, $\beta=k v$.

Based on the above hypothesis analysis and relevant theories of evolutionary game, the following game matrix is constructed, as shown in table 1.

Table 1. Returns matrix of the evolutionary game between government, financial institutions and technology enterprises

\begin{tabular}{|c|c|c|c|}
\hline \multirow{2}{*}{ Government } & \multirow{2}{*}{$\begin{array}{c}\text { Financial } \\
\text { institutions }\end{array}$} & \multicolumn{2}{|c|}{ Science-and-technology enterprise } \\
\hline & & Financing $(p)$ & No financing (1-p) \\
\hline \multirow{2}{*}{$\operatorname{Subsidy}(\lambda)$} & Investment $(x)$ & $\begin{array}{c}R_{g}^{\prime}-\left(\xi+r^{\prime}\right) C_{0},\left(r_{0}+r^{\prime}-\beta\right) C_{0} \\
\left(\alpha-r_{0}-v+\xi\right) C_{0}\end{array}$ & $0,\left(r_{0}+r^{\prime}\right) C_{0}, 0$ \\
\hline & Non-investment $(1-x)$ & $R_{g}{ }^{\prime}-\xi C_{0}, r_{0} C_{0},(\alpha-r-\nu+\xi) C_{0}$ & $0, r_{0} C_{0}, 0$ \\
\hline \multirow{2}{*}{ No subsidy $(1-\lambda)$} & Investment $(x)$ & $R_{g},\left(r_{0}-\beta\right) C_{0},\left(\alpha-r_{0}-v\right) C_{0}$ & $0, r_{0} C_{0}, 0$ \\
\hline & Non-investment $(1-x)$ & $R_{g}, r_{0} C_{0},(\alpha-r-v) C_{0}$ & $0, r_{0} C_{0}, 0$ \\
\hline
\end{tabular}




\section{Stability Analysis and Establishment of System Dynamics Model}

\subsection{The Basic Assumptions of the Model}

According to the return matrix of the three-party evolutionary game of technology finance subjects, the expected net return and average expected return of government departments choosing "subsidy" and "no subsidy" strategies are as follows:

$$
\left\{\begin{array}{l}
E_{\lambda}=x p\left(R_{g}^{\prime}-\left(\xi+r^{\prime}\right) C_{0}\right)+(1-x) p\left(R_{g}^{\prime}-\xi C_{0}\right) \\
E_{1-\lambda}=x p R_{g}+(1-x) p R_{g} \\
\overline{E_{\lambda}}=\lambda E_{\lambda}+(1-\lambda) E_{1-\lambda}
\end{array}\right.
$$

Based on Malthusian model [12], the replicative dynamic equation of government departments' choice of "subsidy" strategy is constructed:

$$
F(\lambda)=\frac{\partial \lambda}{\partial t}=\lambda\left(E_{\lambda}-\bar{E}_{\lambda}\right)=\lambda(1-\lambda) p\left(R g^{\prime}-\xi C_{0}-x r^{\prime} C_{0}-R g\right)
$$

Similarly, if financial institutions choose "investment" and "non-investment" strategies, the expected net risk return and average expected return are respectively:

$$
\left\{\begin{aligned}
E_{x}= & \lambda p\left(r_{0}+r^{\prime}-\beta\right) C_{0}+\lambda(1-p)\left(r_{0}+r^{\prime}\right) C_{0}+(1-\lambda) p\left(r_{0} C_{0}-\beta C_{0}\right) \\
& +(1-\lambda)(1-p) r_{0} C_{0} \\
& E_{1-x}=\lambda p r_{0} C_{0}+\lambda(1-p) r_{0} C_{0}+(1-\lambda) p r_{0} C_{0}+(1-\lambda)(1-p) r_{0} C_{0} \\
\overline{E_{x}}= & x \mathrm{E}_{x}+(1-x) E_{1-x}
\end{aligned}\right.
$$

Based on The Malthusian model, the replication dynamic equation when financial institutions choose the "investment" strategy is constructed:

$$
F(x)=\frac{\partial x}{\partial t}=x(1-x)\left(\lambda r^{\prime} C_{0}-p \beta C_{0}\right)
$$

Similarly, when a technology enterprise chooses the strategy of "financing" and "no financing", the expected net risk return and the average expected return are as follows:

$$
\left\{\begin{aligned}
E_{p}= & \lambda x\left(\alpha-r_{0}-v+\xi\right) C_{0}+\lambda(1-x)(\alpha-r-v+\xi) C_{0} \\
& +(1-\lambda) x\left(\alpha-r_{0}-v\right) C_{0}+(1-\lambda)(1-x)(\alpha-r-v) C_{0} \\
E_{1-p}= & 0 \\
\overline{E_{\mathrm{p}}}= & \mathrm{pE}_{\mathrm{p}}+(1-\mathrm{p}) E_{1-\mathrm{p}}
\end{aligned}\right.
$$

Based on The Malthusian model, the replication dynamic equation when the technology enterprise chooses the "financing" strategy is constructed: 
$F(P)=\frac{\partial P}{\partial t}=P(1-P)\left(\alpha+\lambda \xi-v-x r_{0}-(1-x) r\right) C_{0}$

\subsection{Evolutionary Stability Analysis}

According to Equations (2), (4) and (6), the dynamic system of the government, financial institutions and technology enterprises can be obtained:

$$
\left\{\begin{array}{l}
F(\lambda)=\lambda(1-\lambda) p\left(R g^{\prime}-\xi C_{0}-x r^{\prime} C_{0}-R g\right) \\
F(x)=x(1-x)\left(\lambda r^{\prime} C_{0}-p \beta C_{0}\right) \\
F(P)=P(1-P)\left(\alpha+\lambda \xi-v-x r_{0}-(1-x) r\right) C_{0}
\end{array}\right.
$$

To find the equilibrium point of the three-party evolutionary game, let:

$$
\left\{\begin{array}{l}
F(\lambda)=0 \\
F(x)=0 \\
F(p)=0
\end{array}\right.
$$

Then the equation above has the following eight special equilibrium points on $R=\{(\lambda$, $x, p) \mid 0 \leq \lambda \leq 1,0 \leq x \leq 1,0 \leq p \leq 1\}: E_{1}(0,0,0), E_{2}(0,1,0), E_{3}(0,0,1), E_{4}(1,0,0), E_{5}(1,0,1), E_{6}(1,1,0)$, $E_{7}(0,1,1), E_{8}(1,1,1)$.

According to Lyapunov's first method, the eigenroots of the Jacobian matrix corresponding to the ESS must all be less than 0. The Jacobian matrix $J$ of the tripartite principal dynamical system is shown below.

$$
J=\left[\begin{array}{ccc}
(2 \lambda-1) p A & \lambda(\lambda-1) p r^{\prime} C_{0} & \lambda(\lambda-1) A \\
x(x-1)\left(-p r^{\prime} C_{0}\right) & (2 x-1) p B & x(x-1) B \\
p(p-1)\left(-\xi C_{0}\right) & p(p-1)\left(r_{0} C_{0}-r C_{0}\right) & (2 p-1) C
\end{array}\right]
$$

Including :

$$
\left\{\begin{array}{l}
A=\left(R g-R g^{\prime}+\xi C_{0}+r^{\prime} x C_{0}\right) \\
B=\left(\beta C_{0}-\lambda r^{\prime} C_{0}\right) \\
C=-C_{0}\left(\alpha-\nu+\xi \lambda-r_{0} x+r(x-1)\right)
\end{array}\right.
$$

In this paper, the stability of each equilibrium point is analyzed by eigenroot method. Taking E8 $(1,1,1)$ as an example, the eigenvalues of the jacobian matrix of the system at the equilibrium point are $\lambda_{1}=R_{g}-R_{g}{ }^{\prime}+\xi C_{0}+r^{\prime} C_{0} \quad, \quad \lambda_{2}=\beta C_{0}-r^{\prime} C_{0}$, $\lambda_{3}=-C_{0}\left(\alpha-\nu+\xi-r_{0}\right)$, When condition (3) is satisfied, this point is the asymptotic stable point of the system. Similarly, the analysis results of the other seven equilibrium points are shown in table 2.According to the analysis results, there are three asymptotic stable points, $E_{3}(0,0,1), E_{5}(1,0,1)$ and $E_{8}(1,1,1)$, in this evolutionary game under certain conditions. 
Table 2. Stability analysis of equilibrium solution of Tripartite Evolutionary Game system

\begin{tabular}{|c|c|c|}
\hline Equalization & Eigenvalue symbol & Stability \\
\hline$E_{1}(0,0,0)$ & Positive numbers occur under certain conditions & Instability point \\
\hline$E_{2}(0,1,0)$ & Positive numbers occur under certain conditions & Instability point \\
\hline$E_{3}(0,0,1)$ & $\begin{array}{l}\text { When condition (1) } R g^{\prime}<R g+\xi C_{0} ; \alpha>v+r \text { is met, } \\
\text { both are negative }\end{array}$ & $\begin{array}{l}\text { Asymptotic point } \\
\text { of stability }\end{array}$ \\
\hline$E_{4}(1,0,0)$ & Positive numbers occur under certain conditions & Instability point \\
\hline$E_{5}(1,0,1)$ & $\begin{array}{c}\text { When condition(2) } R_{g}^{\prime}>\xi C_{0}+R_{g} ; r^{\prime}<\beta ; \alpha+\xi>\nu+r \text { is met, } \\
\text { both are negative }\end{array}$ & $\begin{array}{l}\text { Asymptotic point } \\
\text { of stability }\end{array}$ \\
\hline$E_{6}(1,1,0)$ & Positive numbers occur under certain conditions & Instability point \\
\hline$E_{7}(0,1,1)$ & Positive numbers occur under certain conditions & Instability point \\
\hline$E_{8}(1,1,1)$ & $\begin{array}{c}\text { When condition (3) } R_{g}^{\prime}>\xi C_{0}+r^{\prime} C_{0}+R_{g} ; r^{\prime}>\beta ; \alpha+\xi>\nu+r_{0} \text { is } \\
\text { met, both are negative }\end{array}$ & $\begin{array}{l}\text { Asymptotic point } \\
\text { of stability }\end{array}$ \\
\hline
\end{tabular}

\subsection{The System Dynamics Model is Constructed}

In order to further explore the mechanism of tripartite evolutionary game, system dynamics theory is combined with evolutionary game. Based on the game utility function and stability analysis of the three parties in the system, a game flow diagram of system dynamics evolution was constructed, as shown in figure 1.

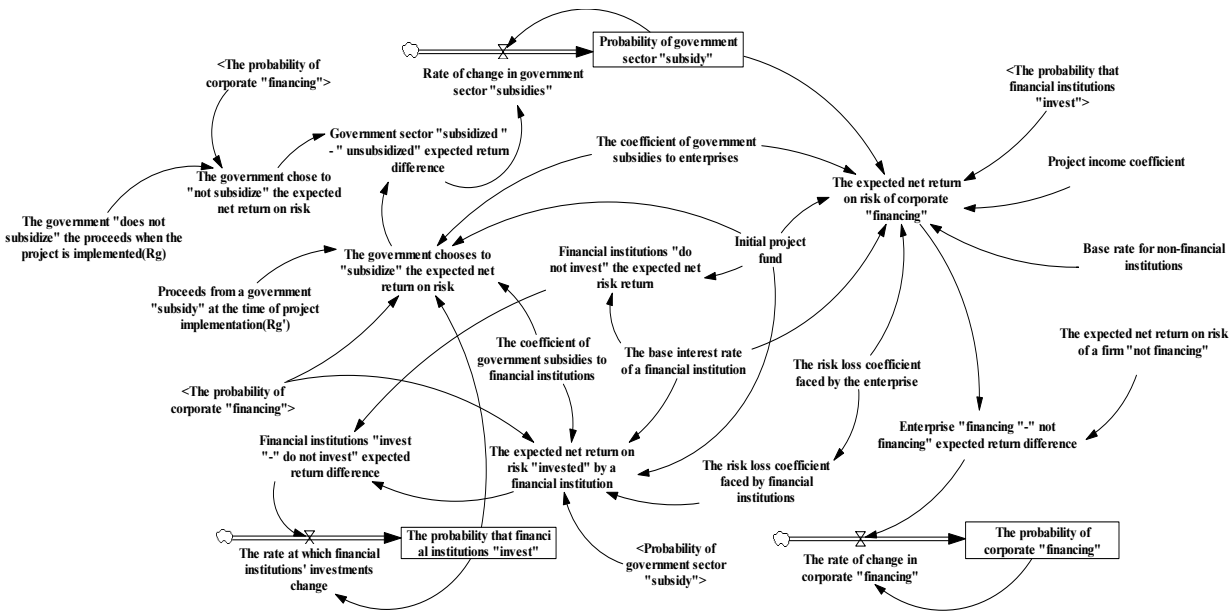

Figure 1. System dynamics flow diagram based on three-party evolutionary game 


\section{SD Simulation and Sensitivity Analysis of Key Parameters}

\subsection{Scenario Simulation and Variable Assignment Description}

In technology finance, $\mathrm{R} \& \mathrm{D}$ projects play an important role in promoting scientific and technological innovation and improving government performance, but their financing difficulties are also typical. In order to facilitate the assignment of parameter variables and better simulation effect, this paper takes the enterprise R\&D project as an example, uses Vensim software to simulate the R\&D project, and conducts sensitivity analysis on key variables in the model. By referring to relevant literature and combining with the actual development of $R \& D$ projects in China, the initial values of variables in the model are as follows:

- (1) Model TIME parameters: Assume INITIAL TIME=0, FINAL TIME=60, TIME STEP=1, and TIME unit Month.

- (2) Initial probability of tripartite subject: $\lambda=0.5, x=0.3, p=0.5$. Basis for assigning values: This paper assumes that government departments and enterprises are risk-neutral, and financial institutions are risk-averse. Therefore, at the initial stage of the model, both the government and enterprises have a half probability to choose whether to promote project implementation, while the probability of financial institutions choosing "investment" is significantly lower than that of the other two parties, which is also consistent with the reality.

- (3)Basic variables: $C_{0}=1, R_{g}=0.2, R_{g}=0.4, r_{0}=6 \%, r=8 \%$. Basis of assignment: In order to simplify the simulation model, the total investment of R\&D project $C_{0}$ was regarded as coefficient 1 ; When enterprises tend to invest in R\&D projects, the income from government subsidy is generally greater than that without government subsidy, because government subsidy will pay some costs, but also reduce the financing difficulty of enterprises to a certain extent, and improve the probability of cooperation between financial institutions and enterprises. Therefore, $R_{g}{ }^{\prime}>R_{g}$ is selected. According to previous literature, the social return rate of enterprise $R \& D$ projects is generally $20 \%-40 \%$, and this paper assumes that all the social benefits are converted into government sector benefits. Refer to the financing cost of related enterprises' R\&D projects and get the interest rate level of financial institutions and non-financial institutions investing in $R \& D$ projects.

- (4)Variation range of key variables: $r=0 \sim 0.03, \quad \xi=0 \sim 0.05, \quad \alpha=0.5 \sim 0.7$, $v=0.1 \sim 0.4, k=0.05, \beta=0.005 \sim 0.02$. Basis for assigning values: This paper assumes that the subsidy coefficient of the government to financial institutions is $0 \sim 3 \%$, and that of the government to enterprises is $0 \sim 5 \%$. The income of an enterprise's R\&D project is divided into private income (enterprise income) and social income. The enterprise net return rate is between $20 \%$ and $40 \%$, so the value of $(\alpha-v)$ should be controlled within 0.4 . The expected risk coefficient $(\beta)$ of financial institutions is jointly determined by the enterprise's expected project risk coefficient $(\alpha)$ and the risk transmission coefficient $(k)$, where the default $k$ is 0.05 . 


\subsection{Stability Point Simulation Analysis}

Based on the stability analysis of the model, three possible stability points $\left(E_{3}, E_{5}, E_{8}\right)$ were simulated by Vensim software. Firstly, the condition of the stable point is calculated by combining variable assignment. It can be seen that at $E_{3}(0,0,1)$, the subsidy coefficient of government to enterprises is far less than 0.2 , which cannot meet $R_{g}{ }^{\prime}<R_{g}+\xi C_{0}$, where $E_{3}$ is not a stable point. Therefore, only two stable points $E_{5}(1,0,1)$ and $E_{8}(1,1,1)$ are numerically simulated in this paper. Secondly, in order to obtain a more obvious simulation effect, this paper assumes that the government adopts a low subsidy strategy at $E_{5}$ stability point and a high subsidy strategy at $E_{8}$ stability point.

- (1) At the stable point $E_{5}(1,0,1)$ simulation, government departments, financial institutions and technology enterprises adopt (subsidy, non-investment, financing) strategy evolution respectively, and the key variables are $r=0.01$, $\xi=0.01, \alpha=0.7, \quad v=0.4, \beta=0.05$. The simulation results are shown in figure 2 .

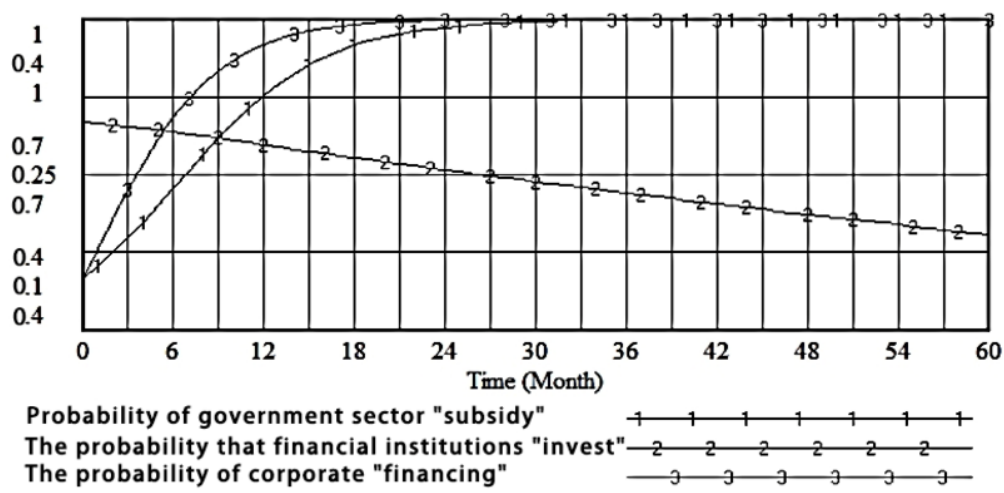

Figure 2. Evolution trajectory of game subject at $E_{5}$ stable point

From above knowable, when the stability conditions $R_{g}^{\prime}-R_{g}>\xi C_{0} ; \alpha+\xi>\nu+r$ is met. Namely in the R\&D project implementation government chooses "subsidies" to earn profits more than government costs, and enterprises get subsidies and expected returns the sum is greater than the risk of loss and the sum of the cost of capital, government and technology enterprises are tended to cooperation, while the financial institutions tend not to cooperation. This shows that when the extra income obtained by the government is high, the government will have a high enthusiasm to implement subsidies. The significant positive externalities of R\&D projects can not only bring performance and reputation to the government, but also directly promote the social and economic development of technology progress. However, when the government subsidy is not enough to compensate for the expected risk losses faced by financial institutions, financial institutions will often choose the "no investment" strategy. Because government subsidies are generally less, technology enterprises pay more attention to the measurement of expected return and risk cost. When the net risk return of R\&D project is greater than 0 , they will choose to obtain project capital from non-financial institutions at a higher cost even if they can not get investment from financial institutions.

- (2) At the stable point $E_{8}(1,1,1)$ simulation, government departments, financial institutions and technology enterprises adopt (subsidy, investment, financing) strategy evolution respectively, and the key variables are $r^{\prime}=0.03, \quad \xi=0.05$, 
$\alpha=0.7, \quad v=0.4, \beta=0.05$. Compared with the stable point $E_{5}$, the government will adopt high subsidy policy. The simulation results are shown in figure 3 .

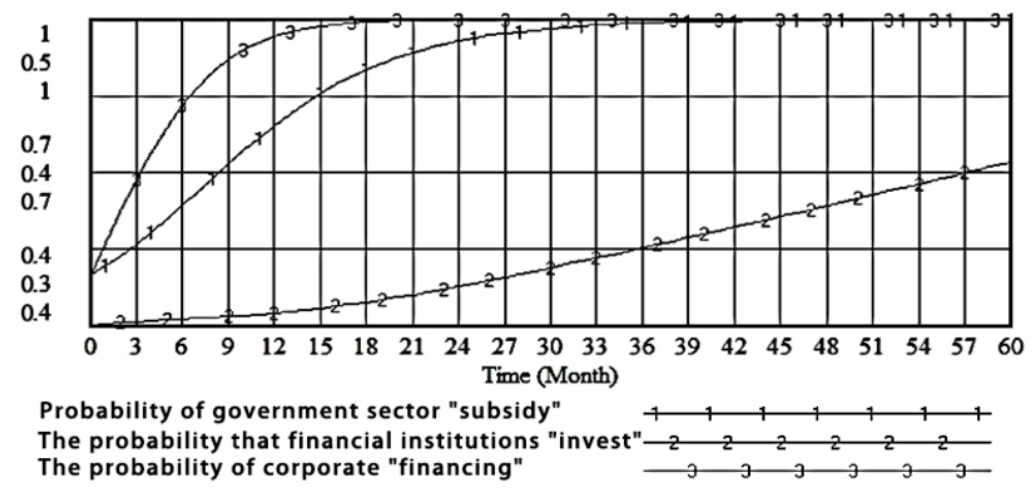

Figure 3. Evolution trajectory of game subject under $E_{8}$ stable point condition

It can be seen from the above figure that only when the stability condition $R_{g}^{\prime}-R_{g}>\xi C_{0}+r^{\prime} C_{0} ; r^{\prime}>\beta ; \alpha+\xi>\nu+r_{0}$ is met, that is, the excess return obtained by the government is greater than the subsidy cost, the net risk return of the enterprise is positive, and the return of financial institutions is greater than the risk loss, can the three parties form a stable win-win cooperation relationship. The comparison with figure 2 shows that when the government increases the subsidy to institutions and enterprises, the evolution time of the government's stabilizing strategy will be prolonged because it will take up more financial expenditure, but the overall impact is small, which also verifies that the government's excess income brought by government subsidies for R\&D projects is greater than the input cost. For financial institutions, when the government chooses high subsidy policy, financial institutions gradually evolve to "investment" strategy because the government subsidy will compensate part of the expected risk loss of financial institutions. However, compared with the government and enterprises, the evolution speed of financial institutions' strategy is obviously slower. This shows that although government subsidies can effectively change the evolution of financial institutions towards "investment" strategy, it is still difficult to fundamentally change the enthusiasm of financial institutions to invest in R\&D projects if only relying on subsidies. For enterprises, when the government chooses high subsidy, it will shorten the evolution time for enterprises to become stable strategy, but the influence is very limited. This also shows once again that if the enterprise expects the net risk return of the project to be positive, in order to achieve the development and profit goals of the enterprise, no matter what kind of subsidy policy the government adopts, in the evolutionary game process, the enterprise will tend to choose project financing.

To sum up, after the simulation analysis of the stability point of the game model, the following conclusions are drawn:

- Conclusion 1: Appropriately increasing the subsidy coefficient by the government will only affect the enthusiasm of government subsidies to a small extent, and the benefits brought by government subsidies are significantly greater than the input costs.

- Conclusion 2: The intensity of government subsidies can only affect the time it takes enterprises to stabilize the strategy to a small extent, and cannot play a decisive role in the choice of financing. 
- Conclusion 3: The intensity of government subsidies has a significant impact on the strategy evolution of financial institutions, but has a weak impact on the strategy evolution speed of financial institutions, indicating that financial institutions still need a long time to change their investment attitude towards R\&D projects even when the government provides high subsidies to financial institutions.

\subsection{Sensitivity Analysis of Key Parameters}

In order to further discuss the influence of key variables in the SD model, the values of key variables $r^{\prime} 、 \xi 、 \alpha, v$ were changed respectively while the basic variables remained unchanged, and the influence of a single key variable on the three-party strategy evolution process was more intuitively analyzed through simulation results. Since $v$ is assumed to be directly proportional to $\beta$, sensitivity analysis of $\beta$ value alone is not carried out in this paper.

\subsubsection{The Coefficient of Government Subsidies to Financial Institutionss}

Based on the above analysis, we know that government subsidies play a significant role in promoting the cooperation between enterprises and financial institutions. To further analyze the influence of government subsidy $\mathrm{R}$ on the three-party strategy evolution, the key variables were assigned as: $\xi=0.05, \alpha=0.7, v=0.4, \beta=0.02$, Assign values to $r^{\prime}$ respectively: $0.01,0.02,0.03$. The simulation results are shown in figure 4 .
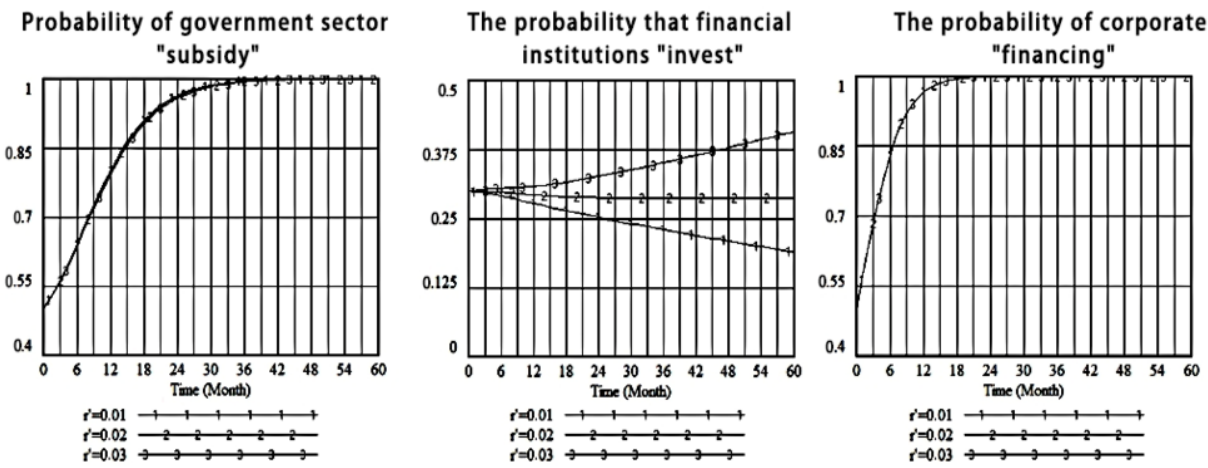

Figure 4. Sensitivity analysis of variable $\left(r^{\prime}\right)$

With the augmentation of the strength of the government subsidies for financial institutions, the enterprise is not affected by the government towards the evolution of the "subsidy" stability time will a little longer, reaching stability strategy of financial institutions from "no investment" gradually to "investment", It shows that the government can effectively change the financial institutions' investment attitude towards R\&D projects by taking appropriate subsidies to financial institutions. 


\subsubsection{The Coefficient of Government Subsidies to Enterprises}

In order to study the influence of government subsidy coefficient $(\xi)$ on three-party strategy evolution, the key variables were assigned $r^{\prime}=0.03, \alpha=0.7, \quad v=0.4, \quad \beta=0.02$, and $\xi$ were assigned $0.01,0.03,0.05$ respectively. The simulation results are shown in figure 5 .
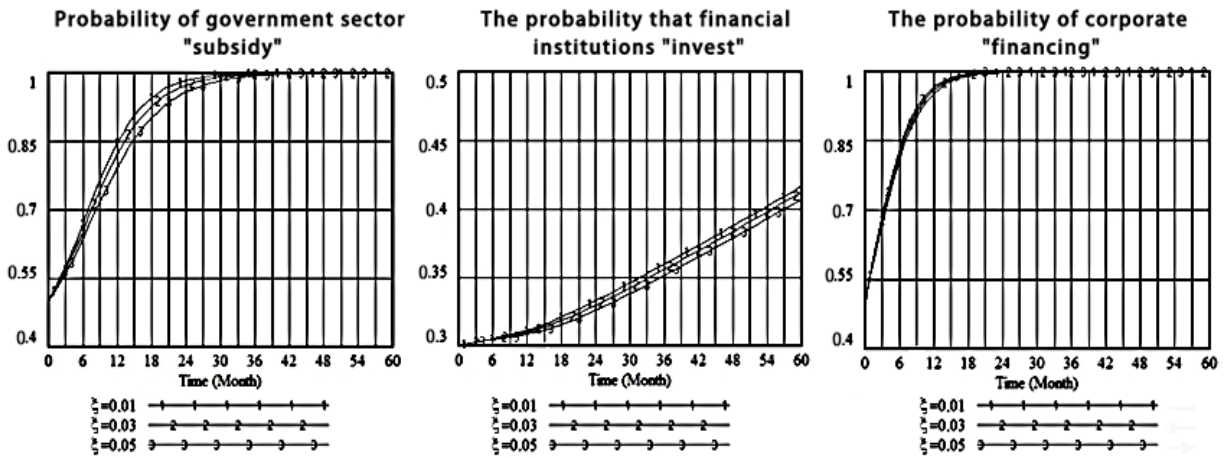

Figure 5. Sensitivity analysis of variables $(\xi)$

The increase of government subsidies to enterprises will shorten the time for enterprises to reach the "financing" strategy stability to a certain extent. The existence of subsidies will make up for the risk loss of some enterprises, so that more enterprises tend to choose the "financing" strategy. At this time, the government needs to invest more financial funds, and the time for the "subsidy" strategy to stabilize has been prolonged. In addition, understanding different, the government improve the strength of subsidies to the enterprise will not raise the initiative "investment" of financial institutions, instead, to a lesser extent to extend its strategy and stable time, that is because of the increase of subsidies will make more enterprises to choose the "financing" strategy, which does not reduce the loss of risk faced by financial institutions. Therefore, in the case of increasing R\&D projects, financial institutions will still "invest" according to the original plan, resulting in a small decrease in the probability of financial institutions choosing "invest" and a corresponding extension of the time for the stability of their "investment" strategy.

\subsubsection{The Income Coefficient of the Enterprise}

Under normal circumstances, the level of enterprise expected earnings will directly affect the enthusiasm of enterprise "financing". The key variables were assigned $r^{\prime}=0.03$, $\xi=0.05, v=0.4, \beta=0.02$, and $\alpha$ were assigned $0.7,0.6,0.5$, respectively. The simulation results were shown in figure 6. 
Probability of government sector "subsidy"
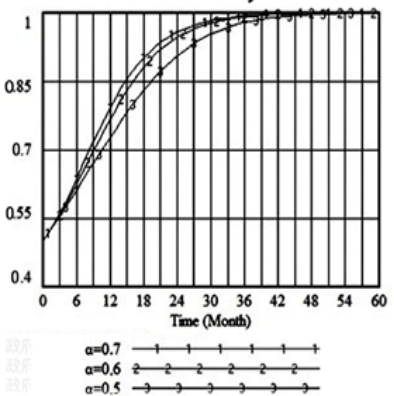

The probability that financial institutions "invest"
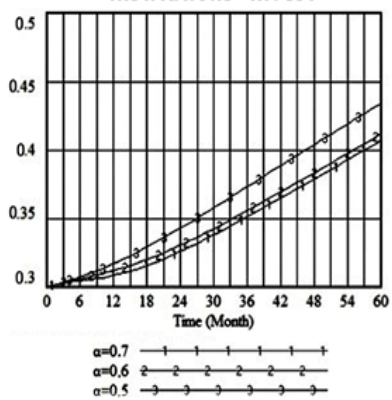

The probability of corporate "financing"

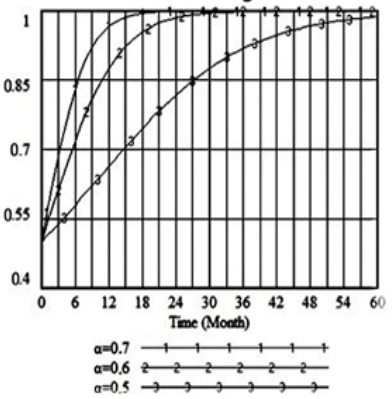

Figure 6. Sensitivity analysis of variables $(\alpha)$

With the decrease of the coefficient of corporate earnings, enterprises "financing" strategy can significantly prolong stability time, in the case of a value also reduce 0.01 , we can see $\alpha=0.5$ the amount of change than the former, that when business is reduced to a certain extent, expected earnings of the larger changes in the stability of the enterprise strategy choices. Secondly, the decrease of the value indicates that the expected net income of the enterprise will decrease. When the income coefficient of the enterprise is lower than the average level of the industry, the enthusiasm of the government will decrease, so the time for the government to choose the "subsidy" strategy will be prolonged. Finally, for financial institutions, low coefficient of corporate earnings, will make the enterprises in the comprehensive consideration the expected net income, choose to give up the R\&D project investment, project quantity also will be reduced to a great extent, stay good projects accounted for more than, therefore, the probability of financial institutions to choose "investment" will also change, the "investment" strategy and stable time will also be shortened accordingly.

\subsubsection{Risk Loss Factor}

The expected net income of $R \& D$ project is not only affected by the income coefficient, but also greatly affected by the risk loss coefficient of the project. In order to further analyze the risk loss coefficient, the key variables are assigned $r^{\prime}=0.03, \xi=0.05$, and $v$ were assigned $0.4,0.3,0.1$. To keep consistent with the previous assumptions, the value of ( $\alpha-v)$ should be controlled within 0.4 , and the corresponding values of $\alpha$ are $0.7,0.7$, and 0.5 .The simulation results are shown in figure 7 .
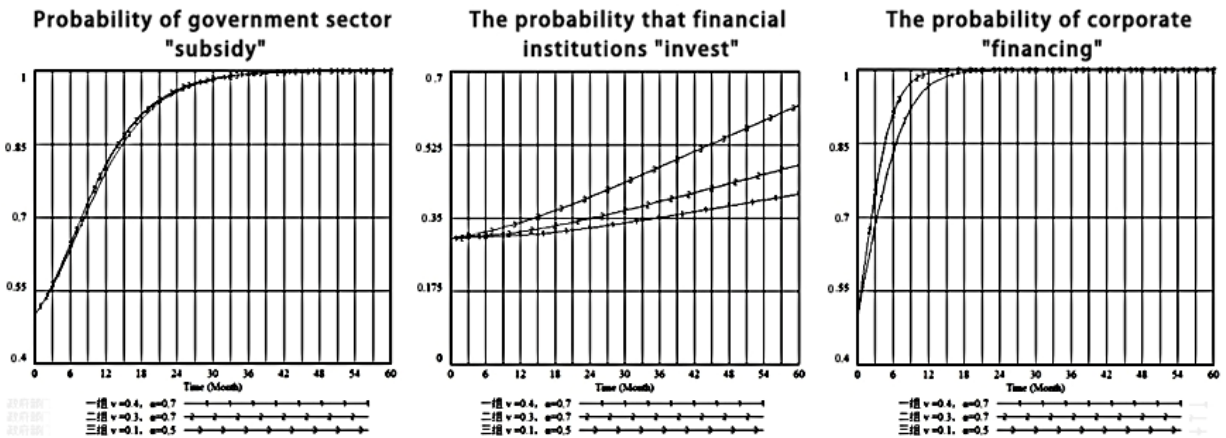

Figure 7.Sensitivity analysis of variables (v) 
As can be seen from the figure above, the risk loss coefficient has the most obvious influence on financial institutions. The control results of group $1(v=0.4, \alpha=0.7)$ and Group $2(v=0.3, \alpha=0.7)$ indicate that under the same expected return coefficient, reducing the risk value will significantly improve the probability of "investment" of financial institutions. If the expected net risk return -, 2 groups $(v=0.3, \alpha=0.7)$ and 3 groups $(v=0.1, \alpha=0.5)$ are considered as the control results, it indicates that in the case of the same expected net risk return, a smaller value will further improve the probability of "investment" of financial institutions. The reason is that under the traditional credit model, the mismatch between risk and return faced by financial institutions is still the root of their low investment enthusiasm. If the risk loss of R\&D projects can be accurately predicted and controlled within the acceptable range of financial institutions, the financing dilemma of technology enterprises will be effectively solved.

Through the initial simulation of the model and sensitivity analysis of key variables, we preliminarily confirmed the three conclusions obtained above, and further obtained the following supplementary conclusions:

- Supplementary conclusion 1: Government subsidy to financial institutions can change financial institutions' investment attitude towards enterprise R\&D projects. Although the evolution of the whole strategy is slow, government subsidy policy is still a good choice to promote win-win cooperation among the three parties.

- Supplementary conclusion 2: The government subsidy coefficient has a very limited impact on the evolution of the strategies of the three parties, while the risk-return coefficient has a significant impact on the "financing" of enterprises.

- Supplementary conclusion 3: Accurate prediction and effective control of enterprise risk loss coefficient is the best choice to solve the financing dilemma of small and medium-sized technology enterprises.

\section{Policy Suggestions}

By constructing the return matrix of the three-party evolutionary game and solving the three-party dynamic replication equation, this paper conducts a progressive analysis on the government departments, financial institutions and technology enterprises in the main body of technology finance, and concludes that there may be two progressive stable points $E_{5}(1,0,1)$ and $E_{7}(1,1,1)$ based on the actual situation. Combined with the system dynamics methodology, the system dynamics flow diagram of the three-party evolutionary game was constructed, and Vensim software was used to conduct numerical simulation on the stable points of the game model. Three basic conclusions were obtained, and sensitivity analysis of key variables was further conducted to supplement the original conclusions. The subsidy coefficient $\left(r^{\prime}\right)$ and risk coefficient $(v)$ of financial institutions from the government play a decisive role in promoting the win-win cooperation among the three parties. At this stage, the domestic financial is still the government-led technology, The government is in the process of implementing its policies, subsidies in addition to the reasonable allocation and adjustment, also should pay attention to the technology risk assessment in the financial system, gradually build the project risk assessment system, and government subsidies into routine examination, improve the transparency of project risk. 
According to the above research results, the policy implications of this paper for promoting win-win cooperation among the three parties of science, technology and finance in China are as follows:

- (1) In the case of technological and financial market failure, the government should make good use of the "tangible hand" and actively play the role of government financial funds in supporting financial institutions. Compared with direct subsidies to enterprises, government subsidies to financial institutions play a more significant role in promoting win-win cooperation among the three parties. At the same time, the government should adjust the subsidy strategy according to the changing market, so that the government subsidy intensity can encourage financial institutions to participate in the technology finance market to the maximum extent.

- (2) Accelerate the construction of enterprise credit system and risk assessment of scientific and technological innovation projects and other technology and financial service platforms, promote data sharing among various parties, so that the government can track the use of subsidy funds, financial institutions can assess the credit risk of enterprises, and the risk value of enterprises' scientific and technological innovation projects can be accurately predicted and assessed. Reduce information asymmetry between government, financial institutions and technology enterprises.

- (3) The development of technology financial rely on government subsidies are not enough, in order to further expand kechuang funding of the project, in addition to financial capital and financial institutions, but also attract social capital into the kechuang domain, build multimodal combined way of financing, through multi-channel financing level technology enterprises financing difficulties; In addition, the government and financial institutions should also focus on the innovation of technology finance mode, and reduce and disperse the risks of technology innovation projects from the source by means of technology business incubator and investment and loan linkage.

- (4) There are different levels of sci-tech finance development in different regions in China, and the degree of coordination between relevant policies and sci-tech finance is not high. Government departments should further improve the policy system according to the development of sci-tech finance in different regions, so as to make it become a booster for the development of sci-tech enterprises; In addition, a strict risk restraint mechanism should be established to avoid potential risks of technology enterprises causing overall risks of technology finance. Government departments should promote mutual support and complementarity between financial institutions and technology enterprises under the condition of controllable risks.

- (5) Technology finance involves multi-disciplinary cross, and the professional talent reserve of government departments and financial institutions is insufficient. Therefore, it is necessary to give full play to the advantages of third-party professional institutions. In key aspects such as project risk assessment and profit prediction, the government and financial institutions can rely on third-party professional institutions to make up for their own shortcomings, enhance the trust among government, enterprise and finance, and form a long-term, stable, cooperative and win-win relationship. 


\section{References}

[1] Wang, Q., Shi, X.: Analysis on the definition, connotation and practice of science and technology finance. Shanghai Finance, 2013, (9), 112-119.

[2] Chen, Z.: International Comparison and Enlightenment of the development of science and technology finance. Scientific Management Research,2018,36(02):92-96.

[3] $\mathrm{Yu}, \mathrm{L} .:$ Research on the contribution of financial support, government and enterprise input to scientific and technological innovation.Science research management, 2015,36(03):57-63.

[4] Li, Y., Chen, W., Liu, S.:Financial Theory \& Practice,2020(12):17-23.

[5] Wang L., Zhou Y., Wang N., Li K.: Research on China's science and technology Finance Support System based on life cycle. Science and Technology Management Research,2020,40(10):36-41.

[6] Long X., CAFS, Jia K.: Financial Institutions and government cooperation technology financial service model research. Economic Research Review, 2015,(7):70-76.

[7] Tan J., Xu J.:Financing Game Analysis between high-tech smes and banks -- A Case study of Nantong Financial Demonstration Zone.Journal of Nanjing University of Technology (Social Science Edition), 2015,(7):70-76.

[8] Yang Y., Xu K., Xu F.: Research on cooperation strategy and Supervision Mechanism of sci-tech finance subject based on Evolutionary game. Science and Technology Management Research, 2018, 38(10):204211.

[9] Yan J., Liu Y., Wan B.: Financial Support for technology-based smes based on life cycle. Finance and Accounting Communications, 2019, (17):14-16.

[10] Gabriel A, Giménez R: Entrepreneurial ignition of the business cycle: The Corporate Finance of Malinvestment. The Review of Austrian Economics,2016,29(3).

[11] Audretsch D B., Lehmann E E, Paleari S, et al: Entrepreneurial finance and technology transfer. The Journal of Technology Transfer,2016,41(1):1-9.

[12] Ritzberger K, Weibull J W: Evolutionary selection in normal form games. Econometrica, 1996(6) :13711399. 